\title{
from the guest editors
}

\section{Jelena Kovačević, Bell Labs, and Martin Vetterli, EPFL, University of California, Berkeley}

\section{Transform Coding: Past, Present, and Future}

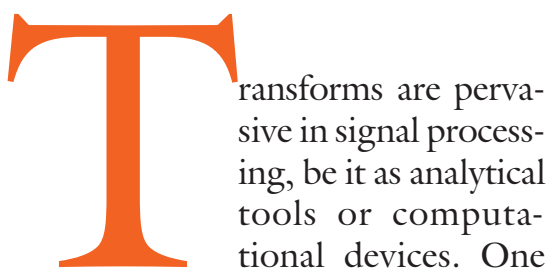
of the most visible successes of transforms and their cousins such as subband coding has been in compression. Both in audio compression, with MP3 making headlines in the popular press, and in image compression, where the discrete cosine transform (DCT) and wavelets are part of the widely used JPEG standards, transforms are at the heart of the compression engine.

At the theoretical level, subband coding, first studied in signal processing for compression, has led to wavelets, a topic with wide ramifications beyond compression and signal processing.

Given the success of transforms in compression, and the flurry of activity in both research and applications around signal processing transforms, it seems appropriate to dedicate a special issue of this Magazine to the topic. The articles in the issue fall into three categories:

$\Delta$ Past: History, background, and theoretical foundation.

- Present: Current applications of transform coding.

$\Delta$ Future: Emerging topics stemming from transform coding and related issues.

Past is covered by the first article in the issue. Vivek K Goyal in "Theoretical Foundations of Transform Coding" reviews what we know about linear expansions and their performance as "compressors" for cer- tain classes of processes. While this is a classic topic with roots in the 1950s and 1960s, with the Karhunen-Loève transform as a classic textbook example, only recently has a fuller understanding been reached (see [13] in the article).

Present is seen through two main areas: technical ideas responsible for both state-of-the-art transform coders as well as for the new image compression standard-JPEG 2000 and the standard itself. In "A Tutorial on Modern Lossy Wavelet Compression: Foundations of JPEG 2000," Bryan E. Usevitch reviews subband and wavelet coding as currently used in image processing standards. $\mathrm{He}$ gives the mathematical background and discusses practical implications. The article leads naturally to the standard that originated from these ideas. JPEG 2000 is covered by Athanassios Skodras, Charilaos Christopoulos, and Touradj Ebrahimi in "The JPEG 2000 Still Image Compression Standard." It is a comprehensive review of the standard, which was recently introduced and includes wavelet decomposition as a key ingredient. Beyond just compression performance, the article points out several system features (such as region of interest) that are enabled by a wavelet decomposition. This also indicates that "raw compression performance" is just one of the important parameters of a real-world standard.

Future, finally, looks at emerging research around transforms and compression, which could lead to better understanding of the problem and new compression techniques. In
"Wavelets, Approximation, and Compression," Martin Vetterli points to the strong interaction between representations such as wavelets, their approximation power (for example, for piecewise smooth functions), and their performance in compression. This subtle interplay is at the heart of the success of wavelets in several applications, including compression and denoising. He also points out that the search for "true" two-dimensional bases is only starting! In "Multiple Description Coding: Compression Meets the Network," Vivek K Goyal tackles some of the issues that already are and will continue to be of outmost importance: the interplay between pure source coding, channel coding, and issues related to transport (reliable or not) across the network. Multiple description coding is one such instance, where the source code has a "network" flavor, that is, it is designed having the transport in mind.

While covering a number of topics, this special issue has no pretense at exhaustiveness. It is noteworthy that, from the theoretical standpoint, the topic of compression using linear transforms concerns not only signal processing, but also information theory, approximation theory, and computational harmonic analysis. Furthermore, complexity issues, circuit implementations, and efficient software are at the heart of applications. This interdisciplinarity is the challenge and beauty of the topic, and we hope to convey both through this special issue. 